\title{
Clinical risk factors and pattern of initial fungal contamination in endoscopic biopsy-derived gastrointestinal cancer organoid culture
}

\author{
Su Youn Nam ${ }^{1}$, Seung Jin Lee ${ }^{1}$, Hee Jung Lim ${ }^{1}$, Ji Young Park ${ }^{2}$, and Seong Woo Jeon ${ }^{1}$
}

\begin{abstract}
${ }^{1}$ Department of Gastroenterology, Center for Gastric Cancer, Kyungpook National University Hospital, Daegu; ${ }^{2}$ Department of Pathology, School of Medicine, Kyungpook National University, Daegu, Korea
\end{abstract}

Background/Aims: Because gastrointestinal tract is not sterile, primary culture has contamination risk despite of massive washing with antimicrobial media. Microbial contamination can play a key role in initial failure during biopsy-derived primary tumor culture.

Methods: Tumor tissue was acquired from esophageal and gastric tumors using endoscopic biopsy. Three-dimensional cultures were performed, and separated spheroids were cultured in media for 7 to 10 days and then transferred to Matrigel (Corning Inc.). We investigated risk factors and patterns of initial fungal contamination.

Results: Initial tumor contamination was observed in $23 \%$ (7/30) of esophageal cancer and $20 \%(3 / 15)$ of gastric cancer samples. Two cases of bacterial contamination occurred during the establishment of culture protocol. Moderate to thick whitish plaques $(p<0.001)$ and food retention in lumen $(p<0.001)$ were risk factors for initial fungal contamination. After exclusion of high risk patients for contamination, no fungal contamination occurred in primary organoid cultures. Fungal contamination was usually detected within 3 days after tumor preparation. However, unusual fungal contamination (GC11 and EC29) was recognized after several passages. Growing spherical shapes resembled cancer organoids. Although they rapidly proliferated and multiple daughter spheroids appeared, the media were translucent. After several passages, yeasts and pseudohyphae were detected on the edges of the solid spherical structures and media.

Conclusions: Moderate to thick whitish plaques and food retention are clinical risk factors for initial fungal contamination during biopsy-derived cancer organoid culture. Most initial fungal contamination was detected within 3 days, but it could be unusually recognized after several passages.

Keywords: Organoids; Gastric cancer; Esophageal cancer; Biopsy; Contamination

\section{INTRODUCTION}

The correlation between primary human tumors and two-dimensional cell line models is low, whereas the similarity between primary three-dimensional (3D) can- cer model and primary human tumors is higher than that between human tumors and mouse models [1]. In vitro $3 \mathrm{D}$ organoid systems are physiologically relevant models [2]. Patient-derived tumor organoids have been generated and investigated for several types of cancer [3- 
5]. Primary tumor tissue can be acquired from surgical specimens or biopsy tissue. The esophagus and stomach are not sterile, with many microbiomes present in the gastrointestinal tract, and thus biopsy tissue can be easily contaminated by bacteria and fungi. The early recognition of contamination is very important, and contaminated discs should be discarded, and the incubator and bench should be cleaned to protect neighboring cultures. Even if bactericidal and fungicidal agents are used and massive washing of tumor tissue is performed during preparation to avoid contamination, initial fungal contamination still occurs, and this initial contamination plays a key role in the failure of primary $3 \mathrm{D}$ culture.

Fungal contamination can manifest with various morphologic patterns, such as yeasts, pseudohyphae, and hyphae. Virulence factor expression varies by fungal morphology [6]. Initial fungal contamination is usually recognized within 3 to 4 days after tissue preparation and is characterized by turbid media. However, we have experienced unusual contamination patterns, leading to failed early recognition of the contamination. Even if methods to avoid contamination during culture and sub-culture have been suggested and applied, little published evidence exists regarding clinical risk factors for initial contamination during endoscopic biopsy-derived primary tumor culture. Here, we evaluated clinical risk factors for initial fungal contamination and also investigated the pattern of initial fungal contamination.

\section{METHODS}

\section{Three-dimensional culture of biopsy-derived prima- ry tumor}

Endoscopic biopsy specimens were acquired from pre-treatment patients with esophageal cancer ( $\mathrm{n}=$ 30) or gastric cancer $(n=15)$ between October 2015 and March 2019 at Kyungpook National University Hospital (Supplementary Table 1). Written informed consent was obtained from each patient. This study was approved by the Institutional Review Board of the Kyungpook National University Hospital (KNUMC 2015-05-212).

Primary 3D cultures were prepared using a modified version of the procedure published by Kondo et al. [7]. Briefly, fresh specimens were transferred to a clean bench and washed with cold Hank's balanced salt solu- tion (HBSS; Invitrogen, Carlsbad, CA, USA). The specimens were chopped into pieces (about $4 \mathrm{~mm}^{3}$ ) using a Feather surgical blade and washed with cold HBSS twice. Tumor fragments were incubated with digestion medium (Dulbecco's modified Eagle medium/F-12 with GlutaMaX, Thermo Fisher Scientific, Waltham, MA, USA), Liberase Dispase-High (Roche Applied Science, Mannheim, Germany), and antibiotic-antimycotic at $37^{\circ} \mathrm{C}$ for 1 hour. The digested specimens were sequentially filtered through a $100 \mu \mathrm{m}$ cell strainer (BD Bioscience, Franklin Lakes, NJ, USA) and a $40 \mu \mathrm{m}$ cell strainer (BD Bioscience, Franklin Lakes, NJ, USA). The fragments remaining on the cell strainer were collected and cultured in stem cell medium (StemPro hESC SFM, Thermo Fisher Scientific) supplemented with basic fibroblast growth factor (Invitrogen), antibiotic-antimycotic, and 2-mercaptoethanol (Sigma-Aldrich, St. Louis, MO, USA) in a non-treated culture dish (SPL, Pocheon, Korea). To prevent bacterial and fungal contamination, we added penicillin, streptomycin, and antimycotics (pen/strep plus amphotericin B) to media

\section{Primary organoid culture and passage}

Spheroids were embedded in a Matrigel matrix (Corning Inc., Corning, NY, USA) mixed with HBSS at a 3:2 ratio and incubated at $37^{\circ} \mathrm{C}$ in a $5 \% \mathrm{CO}_{2}$ humidified incubator for 30 minutes. Then $500 \mu \mathrm{L}$ of stem cell medium was added into the culture dish. The culture medium was changed regularly, and passages were carried out for spheroids larger than $1,000 \mu \mathrm{m}$.

\section{Fungal plaque covering on primary tumor}

Whitish fungal plaques frequently covered tumor or normal mucosa in esophageal cancer. We classified primary tumor by fungal plaques thickness (Fig. 1). No plaque (Fig. 1A), thin whitish plaque (Fig. 1B), moderate plaque (Fig. ${ }_{1}$ ), thick plaque (Fig. $1 \mathrm{D}$ ), and very thick plaque (Fig. 1E) covered primary tumors.

\section{Statistical analysis}

Fisher's exact test were performed for the categorical data and $t$ test was performed for the continuous variables. We used STATA version 12 (StataCorp., College Station, TX, USA) for all analyses. All statistical tests were two sided, and $p$ values less than 0.05 were considered statistically significant. 


\section{KJIM}

The Korean Journal of Internal Medicine Vol. 36, No. 4, July 2021

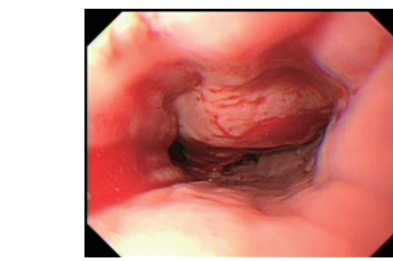

A

Without plaque (EC23)

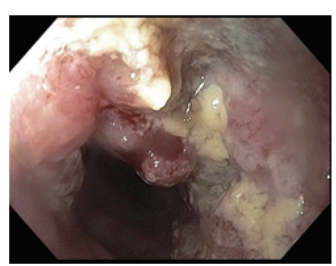

Moderate plaque (EC7)

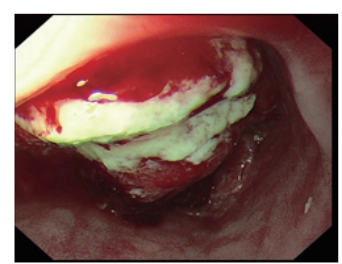

E

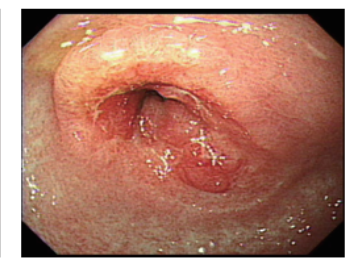

Without plaque (GC1)

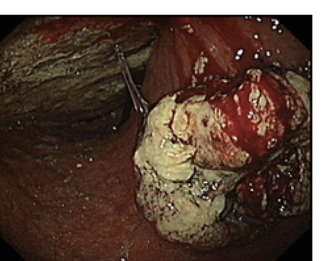

Very thick plaque + food (GC14)

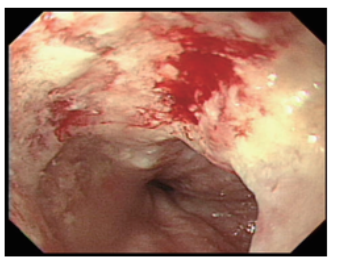

Thin plaque (EC19)

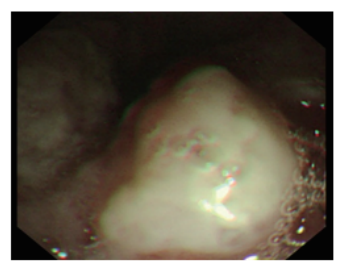

Thick plaque (EC21)

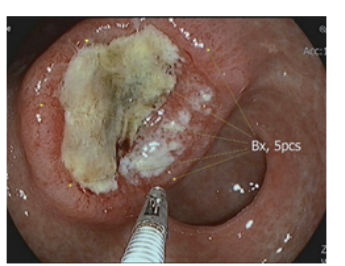

Necrotic ulcer base/biopsy at ulcer margin (GC15, GC9)

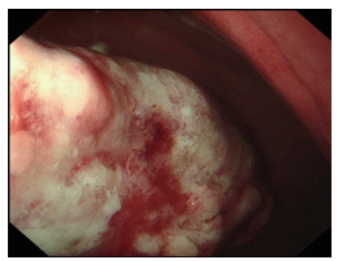

Thin plaque (GC7)

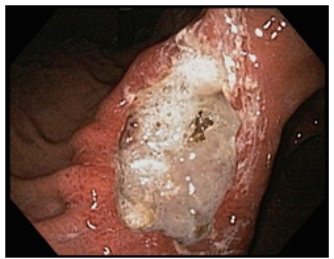

Thick plaque (GC11)

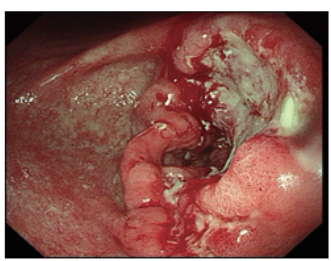

$\mathbf{F}$

Figure 1. Endoscopic features of contaminated and non-contaminated organoid. (A) No whish plaque was noted on the primary tumor. (B) Primary tumor was covered with film like thin whitish plaque. (C) Primary tumor was covered with moderate whitish plaques. (D) Primary tumor was covered with thick plaques. (E) Primary tumor was covered with very thick whitish plaques with or without luminal food material in contaminated primary organoids. (F) Primary tumor showed necrotic ulcer base without plaques and biopsy was done at non-necrotic ulcer margin. EC, esophageal cancer; GC gastric cancer.

\section{RESULTS}

\section{Contamination rate in biopsy-derived esophageal and gastric cancer culture}

Mean age of enrolled patients was 64.9 years and most persons (93.3\%) are men (Supplementary Table 1). Initial contamination was observed in $23 \%$ (7/30) of esophageal cancer and $20 \%(3 / 15)$ of gastric cancer tissue samples (Table 1). Former two cases were contaminated by bacteria and later five cases of esophageal cancer cultures were contaminated by yeast. After the establishment of a bacterial-contamination free protocol, gastric cancer organoid culture was performed. Therefore, bacterial contamination was not observed during gastric cancer organoid culture preparation. Most initial contamination was detected on day 3 , but the recognition of contamination was delayed in two cases (EC29 and GC11) (Table 1). We analyzed demographic and endoscopic factors between eight fungal contaminated cases and 35 non-contaminated cases.

\section{Endoscopic features of initial fungal contaminated organoid}

Almost cases of initial fungal organoid contamination, showed tumor covered with moderate to thick whitish plaques during endoscopic biopsy (Table 1 and Fig. $1 \mathrm{C}-$ ${ }_{1 \mathrm{E}) .} \mathrm{EC}_{15}$ showed a clean base on the primary tumor, and yeast contamination was due to a handling error at bench. The tumor base was clean or only covered with thin exudates without thick whitish plaques in non-contaminated organoids (Fig. $1 \mathrm{~A}$ and $1 \mathrm{~B}$ ).

\section{Risk factors for initial fungal contamination}

Age, sex, diabetes, hypertension, heart disease, smoking status, and drinking status had no association with initial fungal contamination (Table 2). Initial fungal 
Table 1. Initial contamination pattern in biopsy-derived gastrointestinal organoid and clinical feature

\begin{tabular}{|c|c|c|c|c|c|}
\hline & $\begin{array}{l}\text { Detection time of } \\
\text { contamination }\end{array}$ & $\begin{array}{c}\text { Contamination } \\
\text { organism }\end{array}$ & $\begin{array}{c}\text { Contamination } \\
\text { pattern }\end{array}$ & Endoscopic findings & Cell type \\
\hline $\mathrm{EC} 1$ & Day 3 & Bacteria & Typical & Clean tumor base & SQC \\
\hline EC6 & Day 3 & Bacteria & Typical & Minimal whitish plaque & SQC \\
\hline $\mathrm{EC}_{7}$ & Day 3 & Fungus & Typical & Moderate whitish plaques & SQC \\
\hline EC9 & Day 6 & Fungus & Typical & Food \& minimal whitish plaques & SQC \\
\hline $\mathrm{EC}_{15}$ & Day 6 & Fungus & Typical & Without whitish plaque & SQC \\
\hline $\mathrm{EC} 21$ & Day 3 & Fungus & Typical & Thick whitish plaques & SQC \\
\hline $\mathrm{EC} 29$ & 2 Months & Fungus & Unusual & Very thick whitish plaques & SQC \\
\hline $\mathrm{GC}_{5}$ & Day 3 & Fungus & Typical & Food \& whitish plaques & $\mathrm{ADC}, \mathrm{PD}$ \\
\hline GCi1 & 3 Months & Fungus & Unusual & Thick whitish plaques & $\mathrm{SRC}$ \\
\hline $\mathrm{GCl}_{4}$ & Day 3 & Fungus & Typical & Food \& very thick whitish plaques & $\mathrm{ADC}, \mathrm{MD}$ \\
\hline
\end{tabular}

EC, esophageal cancer; SQC, squamous cell carcinoma; GC gastric cancer; ADC, adenocarcinoma; PD, poorly differentiation; SRC, signet ring cell; MD, moderate differentiation.

Table 2. Demographic factors of initial fungal contamination during biopsy-derived primary organoid culture

\begin{tabular}{lccc}
\hline Variable & $\begin{array}{c}\text { Non- } \\
\text { contamination } \\
(\mathrm{n}=35)\end{array}$ & $\begin{array}{c}\text { Fungal } \\
\text { contamination } \\
(\mathrm{n}=8)\end{array}$ & p value ${ }^{\mathrm{a}}$ \\
\hline Age, yr & $66.1 \pm 10.4$ & $62.1 \pm 10.9$ & 0.338 \\
Male sex & $33(94)$ & $7(88)$ & 0.497 \\
Diabetes & $7(20)$ & $1(13)$ & 0.623 \\
Hypertension & $9(26)$ & 0 & 0.107 \\
Heart disease & $1(3)$ & 0 & 0.629 \\
Current smoker & $18(51)$ & $4(50)$ & 0.942 \\
Current drinker & $21(60)$ & $5(63)$ & 0.896 \\
\hline
\end{tabular}

Values are presented as mean \pm SD or number (\%).

${ }^{a} p$ values were derived from $t$ test or Fisher's exact test.

contamination rarely occurred in tumor tissue without whitish plaques $(5 \%, 1 / 19)$, but initial fungal contamination always occurred in tumor tissue covered with moderate to thick whitish plaques $(100 \%, 5 / 5)$ (Table 3). Biopsy was performed on the ulcer margin in cases of tumor with ulceration and fungal contamination did not occur in such cases (Fig. 1F). Food retention in the lumen was associated with fungal contamination (100\% in cases of food retention vs. $14 \%$ in cases of tumors without food retention, $p<0.001$ ) (Table 3 ). In summary, moderate to thick whitish plaques on the tumor and food retention in the lumen were important risk factors for initial fun- gal contamination.

\section{Morphology of usual fungal contamination}

Fungal contamination was detected on 3 days after primary organoid preparation in $\mathrm{EC}_{7}, \mathrm{EC}_{21}, \mathrm{GC}_{5}$, and $\mathrm{GC}_{14}$ (Table 1). Fungal contamination was detected as yeasts on day 6 in tumor with minimal plaque (EC9) or without whitish plaque (EC15) (Fig. 2A). Massive fungal contamination was detected as yeasts and psueohyphae in organoid and turbid media on day 3 in tumor covered with moderate whitish plaques (EC7) or thick whitish plaque (EC21) (Fig. 2B). For gastric cancer, fungal contamination was detected with increased turbidity on day 3 in cases of tumor covered with whitish plaques and food retention in the lumen (GC5 and GC14) (Fig. 2C). Massive washing using antibacterial/antifungal media could not save contaminated organoids.

\section{Morphology of media fungal contamination with preserved organoid}

Media fungal contamination with preserved organoid was detected on day 8 in EC27 (Fig. $3 \mathrm{~A}$ and $3 \mathrm{~B}$ ). Yeast, psuedohyphae, and hypahae were observed mainly in translucent media. After massive washing with antibacterial/antifungal media, organoids were implanted on Matrigel. They grew well after multiple passages (Fig. 3C). 
Table 3. Endoscopic factors of initial fungal contamination during biopsy-derived primary organoid culture

\begin{tabular}{|c|c|c|c|}
\hline Variable & $\begin{array}{l}\text { Non-contamination } \\
(\mathrm{n}=35)\end{array}$ & $\begin{array}{l}\text { Fungal contamination } \\
\qquad(\mathrm{n}=8)\end{array}$ & $p$ value $^{\mathrm{a}}$ \\
\hline \multicolumn{4}{|c|}{ Whitish plaques/necrotic ulcer on primary tumor } \\
\hline Without whitish plaque & $18(95)$ & $1(5)$ & $<0.001$ \\
\hline Thin whitish plaques & $3(60)$ & $2(40)$ & \\
\hline Moderate to thick whitish plaques & 0 & $5(100)$ & \\
\hline Necrotic ulcer/biopsy at ulcer margin & $14(100)$ & o & \\
\hline \multicolumn{4}{|l|}{ Food retention in lumen } \\
\hline Food retention $(-)$ & $35(86)$ & $5(14)$ & $<0.001$ \\
\hline Food retention $(+)$ & o & $3(100)$ & \\
\hline \multicolumn{4}{|l|}{ Overall endoscopic factors } \\
\hline Low endoscopic risk & $35(100)$ & $1(12)$ & $<0.001$ \\
\hline High endoscopic risk & 0 & $7(88)$ & \\
\hline
\end{tabular}

Values are presented as number (\%). Low endoscopic risk refers to no or thin whitish plaque on primary tumor and food retention in lumen. High endoscopic risk refers to moderate to thick whitish plaques on primary tumor or food retention in lumen. ${ }^{\mathrm{a}} \mathrm{p}$ values were derived from Fisher's exact test.

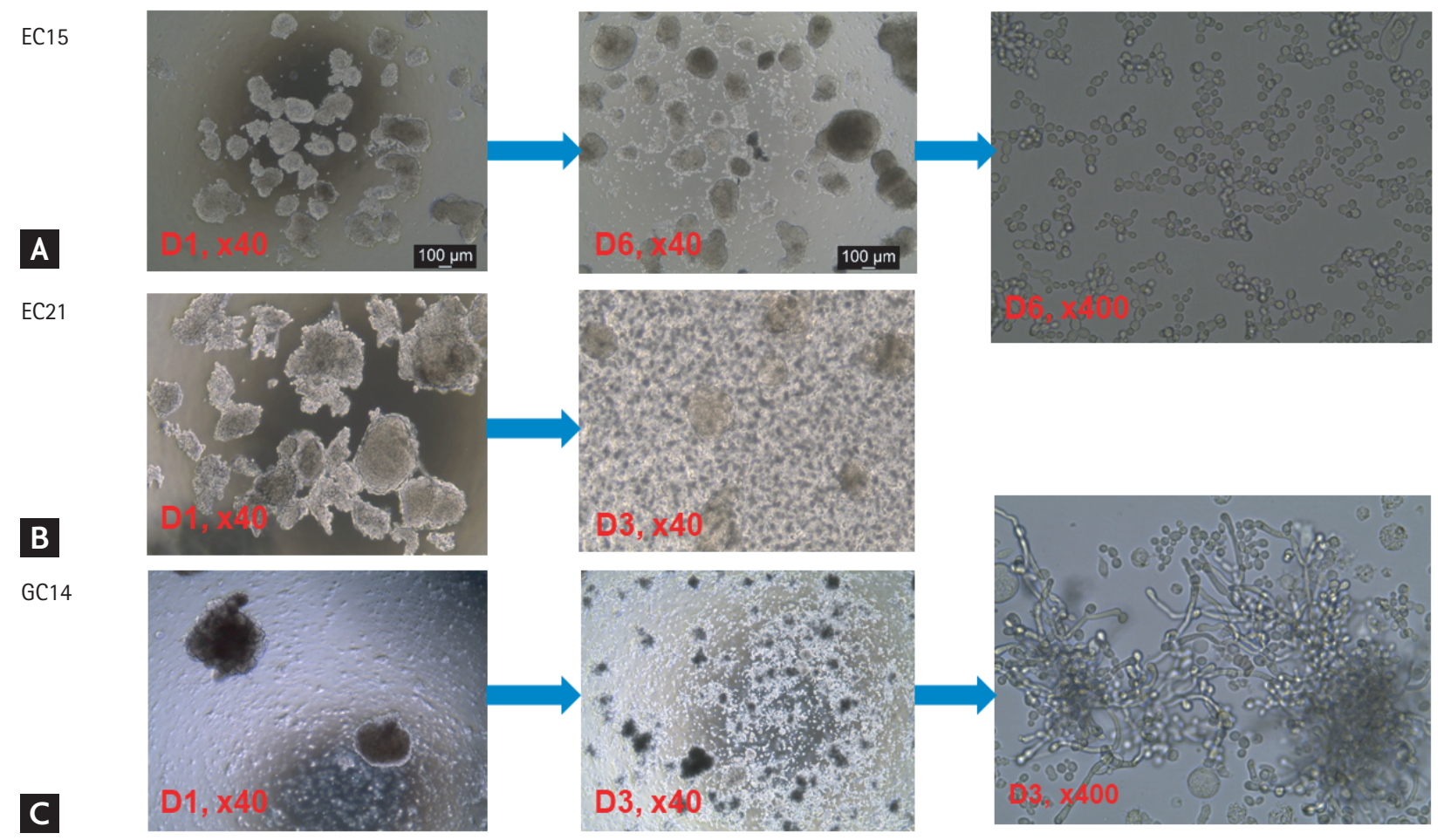

Figure 2. Usual pattern of initial fungal contamination. (A) Initial fungal contamination on tumor (EC15). Clean base tumor on endoscopy. Round yeast cells were detected on day 6. (B) Initial fungal contamination on tumor (EC21). Tumor was covered with thick whitish plaques. Massive fungal contamination was detected on day 3. (C) Initial fungal contamination on tumor (GC14). Tumor was covered with thick whitish plaques and a large amount of food material was observed in the stomach. Round yeast cells, pseudohyphae, and hyphae were observed on day 3 . 


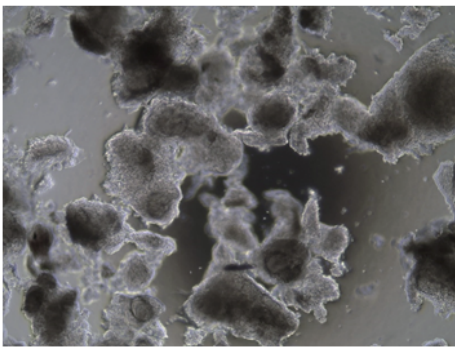

D1, $\times 40$

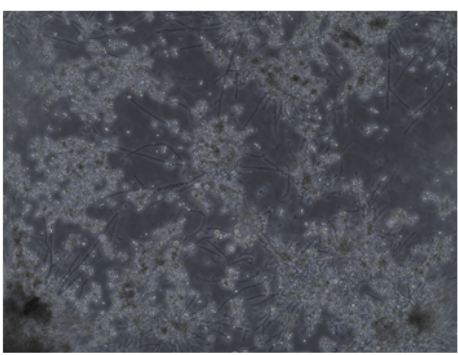

D8, media, 40x

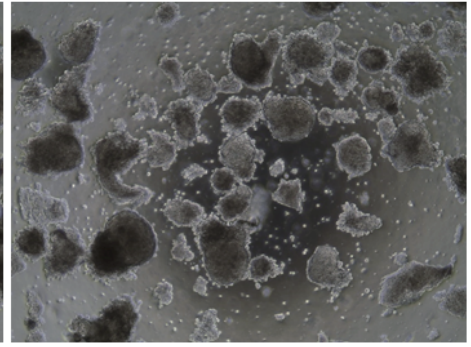

D4, $\times 40$

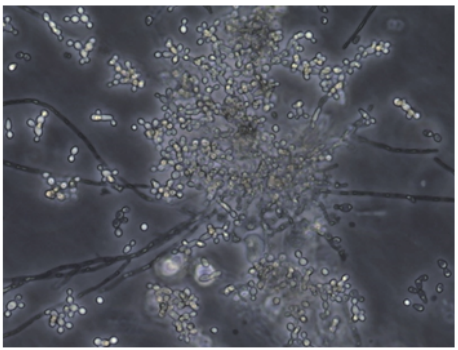

D8, media, 400x

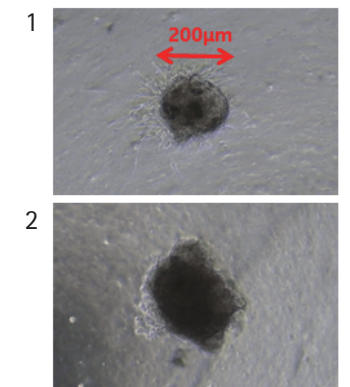

3

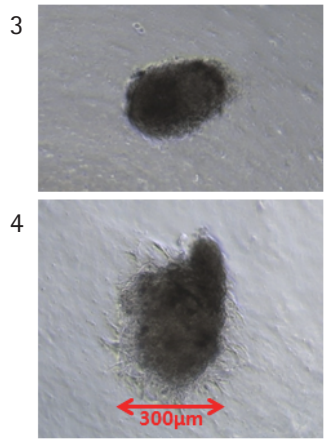

C

D8 (P1-D1)
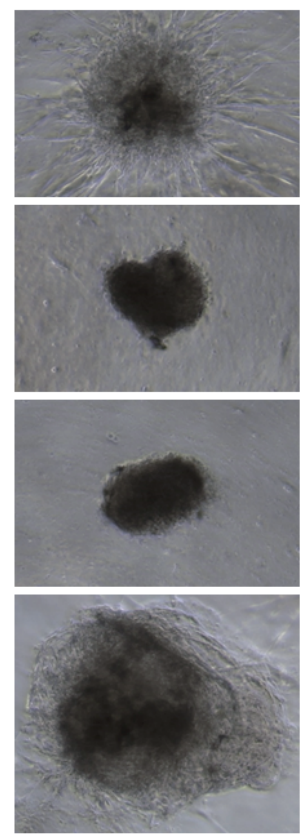

D19 (P1-D12)

Figure 3. Media contamination with preserved organoid (EC27). (A) Primary tumors were plated in round disc (day 1). Small dots were noted in media (day 4). (B) Media fungal contamination with preserved organoid was detected on day 8 . Yeast, psuedohyphae, and hypahae were observed in media. (C) After thorough washing, organoid was implanted on Matrigel(Corning Inc.) (D8, P1-D1). Good growth was observed after passaging. P1 refers to the first seeding on Matrigel.

\section{Morphology of unusual fungal contamination}

Solid spheroid formation was observed in the media after 3D culture preparation (GC11) (Fig. 4A). After seeding on Matrigel, spherical shapes grew like cancer organoids and they maintained clear edges and thick structure. Although organoid structures extraordinarily quickly proliferated and multiple daughter spheroids appeared in the Matrigel, the medium remained translucent (Fig. $4 \mathrm{~B})$. After the second seeding, small organoid structures rapidly proliferated with a bizarre pattern, but the black circular shape was preserved. We suspected contamination, but the medium was still translucent. We found aggregated small round structures on high-power field images (200x) during a retrospective review (Fig. 4C). After the third seeding, round solid structures rapidly proliferated, with easy fragmentation by needle and numerous daughter structures (Fig. 4D). After the fourth seeding, we observed amorphous proliferation with easy fragmentation by touch and numerous daughter structures. Small round yeasts and pseudohyphae were detected from both solid spherical structures and the me- dium in a high-power field (Fig. 4E). The EC29 growing pattern was similar to that of the 3D structure of GC11 (data not shown).

\section{Hematoxylin and eosin staining of primary cancer organoid}

Hematoxylin and eosin staining of well-growing esophageal cancer organoid (EC19) revealed a solid round structure in low-power field microscopy and squamous cell carcinoma with a high-power field (Fig. $5 \mathrm{~A}$ ). Numerous fungal pseudohypae and yeasts were present in fibrinous materials in contaminated esophageal cancer organoid (EC29) (Fig. 5B).

\section{Outcome after exclusion of high risk patients}

No fungal contamination occurred in primary organoid cultures (EC3O-EC35 and GC15-GC21), after we excluded the patients whose primary tumors are covered with moderate to thick whitish plaques and those in whom luminal food retention is observed. 


\section{KJIM'}

A

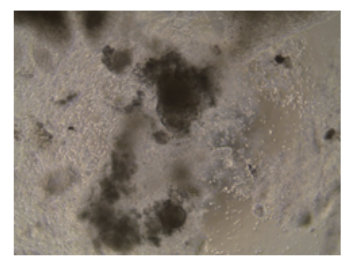

P0-D1

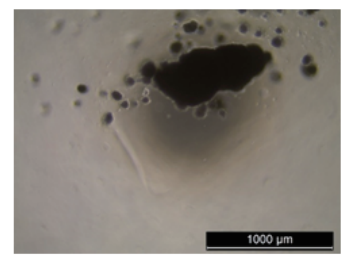

B P1-D3

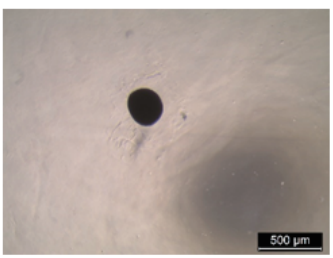

P2-D1

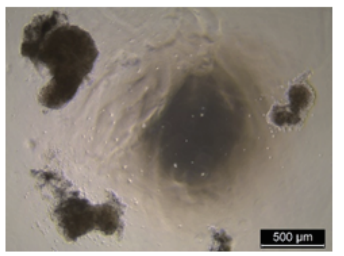

P0-D5

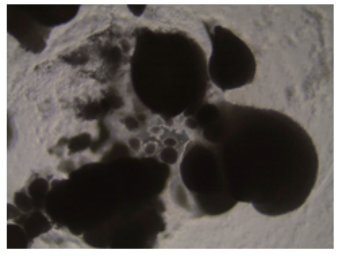

P0-D43

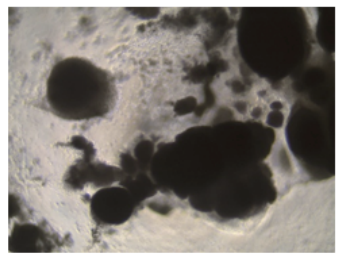

P2-D8

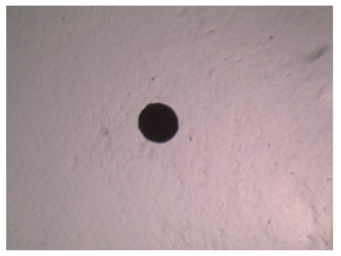

D

P3-D1

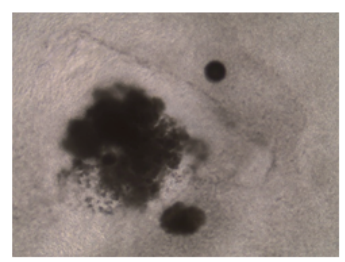

P4-D1

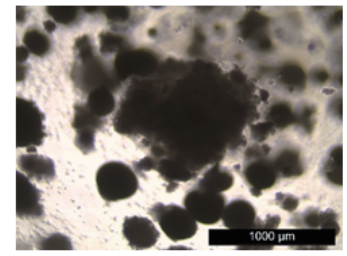

P3-D20

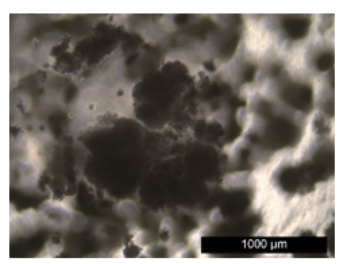

P4-D9

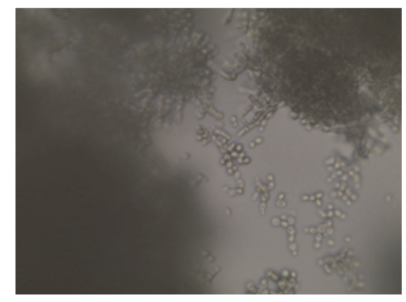

$400 x$

Figure 4. Unusual initial fungal contamination in gastric cancer organoid (GC11). (A) Organoid formation was observed in the medium. (B) After seeding on Matrigel (Corning Inc.), organoid structures extraordinarily rapidly proliferated, and very thick structure formation was observed. (C) After the second seeding, small organoid structure was rapidly proliferated with a bizarre pattern. The black circular shape was preserved. Researchers found aggregated small round structures on high-power field images (200x) during retrospective review. (D) After the third seeding, the round solid structure was rapidly proliferated with fragmentation and numerous daughter structures. We suspected contamination, but the medium remained clear, so we performed hematoxylin and eosin staining to investigate the bizarre proliferation pattern. (E) After the fourth seeding, amorphous proliferation with numerous daughter structures was observed. Small round yeast cells and pseudohyphae were observed under high-power magnification (400x).

A

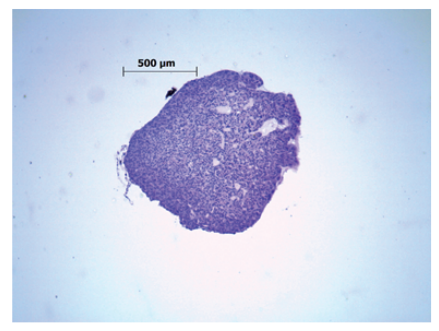

$40 \mathrm{x}$

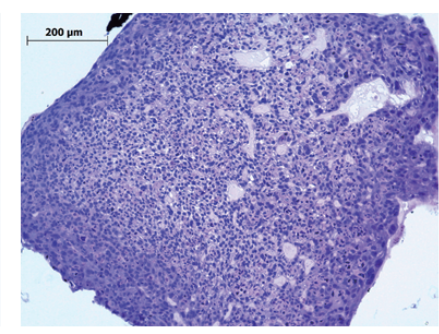

$100 x$

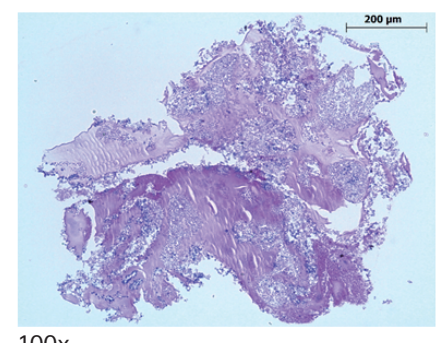

$100 x$

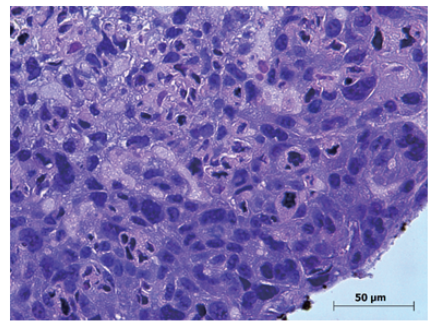

$400 x$

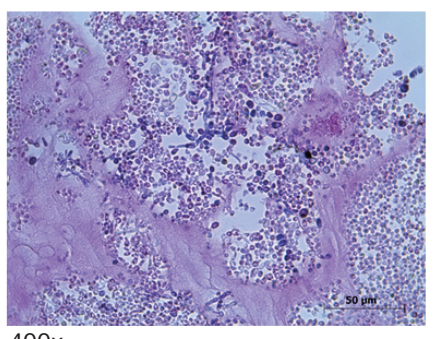

$400 x$

Figure 5. Hematoxylin and eosin staining of non-contaminated and contaminated esophageal cancer organoid. (A) Hematoxylin and eosin staining of a well-growing esophageal cancer organoid (EC19) showed a solid round structure in low-power field microscopy and squamous cell carcinoma in the high-power field. (B) Numerous fungal pseudohyphae and yeasts present in fibrinous materials in contaminated esophageal cancer organoid (EC29). 


\section{DISCUSSION}

Moderate to thick whitish plaques on the tumor and food retention in the lumen were risk factors for initial fungal contamination during biopsy-derived primary tumor 3D-culture. After exclusion of high risk patients, any bacterial or fungal contamination did not occur in biopsy derived organoid cultures. Fungal contamination was usually detected within 3 to 6 days after tumor preparation. However, unusual fungal contamination was recognized after several passages, featuring bizarre morphology, extraordinarily rapid proliferation, and compact spherical 3D structure resembling cancer organoids maintaining clear edges in translucent medium. These unusual features led to late detection of fungal contamination. Demographic factors had no association with initial fungal contamination.

Sterile cell culture is vitally important to avoid contamination by microorganisms, which could interrupt the integrity of cell system and ruin experiments. Unfortunately, culture conditions, such as incubator temperature, humidity, and nutrient content in media, promote the growth of microbial contaminants, which grow rapidly and easily spread to neighboring culture dishes. Therefore, it is important to recognize potential contaminants and sources of infection. Because the esophagus and stomach are not sterile and many microbiomes coexist in the gastrointestinal tract, biopsy-derived primary culture is exposed to contamination risk by bacteria and fungi. In this study, initial overall contamination was observed in $24 \%$ of esophageal cancer cultures and $21 \%$ of gastric cancer cultures. Early two cases were contaminated by bacteria and later five cases of esophageal cancer organoids were contaminated by fungi. Bacterial contamination did not occur after the $3 \mathrm{D}$ culture protocol was established. Bacterial contamination can be avoided through careful handling and the addition of pen/strep to the media. Even though we washed tumor tissue during initial preparation and used antibiotic-antimycotic (pen/strep plus amphotericin B) media, we still experienced initial fungal contamination. This initial fungal contamination was a major cause of primary organoid culture failure. Therefore, the control of initial fungal contamination can be key to increasing the yield of primary culture.

In this study, moderate to thick whitish plaques and food material in the lumen were important clinical risk factors for initial fungal contamination during biopsy-derived gastrointestinal cancer $3 \mathrm{D}$ culture. Initial fungal contamination rarely occurred in tumors without whitish plaques (5\%), but it always occurred in tumor covered with moderate to thick whitish plaques (100\%). Massive washing using pen/strep/amphotericin B contained media can't overcome fungal contamination in cases of primary tumors heavily contaminated with fungi in vivo. We acquired tissue from ulcer margin in cases of tumors with ulcer exudates, and fungal contamination did not occur in such cases. Food retention in the lumen was also associated with fungal contamination. Fungal contamination occurred in 100\% of cases associated with food retention and in $14 \%$ of tumors without food retention. These results suggest that we should exclude the patients whose primary tumors are covered with moderate to thick whitish plaques or those in whom luminal food retention is observed, to avoid fungal contamination and to increase the yield of primary tumor 3D culture. Actually, fungal contamination did not occur in biopsy derived organoid cultures after exclusion of high risk patients. Even though methods to avoid contamination during cell culture have been applied, there is little published evidence regarding the clinical risk factors for contamination during biopsy-derived primary $3 \mathrm{D}$ culture. To our knowledge, this study is the first study to investigate the clinical risk factors for initial fungal contamination during biopsy-derived primary 3D culture from non-sterile gastrointestinal tract.

Initial fungal contamination was detected as in the form of yeasts and pseudohyphae in turbid media within 3 to 6 days after primary tumor preparation in most cases. This typical contamination can be detected easily during culture. Massive washing couldn't save the contaminated organoids and all those contaminated disc was discarded. However, mild media contamination with preserved organoid can be salvaged. After thorough washing, organoids (EC27) were implanted on Matrigel, and they grew well after multiple passages. Most fungal contaminants are clearly visible with turbid media and fungus such as yeast, pseudohyphae, or hyphae. However, two cases of $3 \mathrm{D}$ culture (one esophageal cancer and one gastric cancer) showed unusual fungal contamination patterns. Spheroid formation was observed in the media after $3 \mathrm{D}$ culture preparation. Although extraordi- 
narily rapid proliferation and multiple daughter spheroids appeared in the Matrigel, they maintained thick solid structures and the media remained translucent. The growing spherical shapes resembled cancer organoids and maintained their clear edges. We suspected contamination, but those unusual conditions led to late detection of fungal contamination. Amorphous proliferation with touch fragmentation by needle and numerous daughter structures were also observed. After several passages, small round yeasts and pseudohyphae were detected on the edges of solid spherical structures and in the media through high-power microscopy.

Many fungal species have the ability to change the morphology; yeast, psueophyphae, or hyphae and such morphological changes are associated with virulence in several human fungal pathogens $[8,9]$. Yeasts are single ovoid cells that may show budding patterns, whereas pseudohyphae and hyphae are filamentous [10]. The fungal species that most commonly infects the upper gastrointestinal tract is Candida albicans. In the commensal state, the yeast form colonizes on the mucosal surface, wherein the hyphal form allows for nutrient scavenging, rapid attachment, and biofilm formation [11]. During a disseminated infection, the hyphal form plays an important role in the lysis of neutrophils and macrophages, biofilm formation, and immune evasion [12,13]. Virulence gene expression in Candida species increase according to yeast-pseudohyphal-hyphal morphology [6]. In EC29 and GC11, yeasts were confined within the thick spherical solid structure with clear edges and media was translucent during several passages. And then evolution of psuedohyphae and release of yeasts from dense solid structures were detected in turbid media. During the retrospective review, we found a photograph captured yeast forms on the edges of spherical structure, which we missed. In this unusual contamination pattern, the translucent medium and solid thick structure with minimal release of fungal cells from the solid structure led to late detection of contamination. Translucency of a culture medium may be due to low release of virulence factors from the yeast form.

A report from major cell banks showed that $39 \%$ of cell lines were contaminated and major contaminating microbes were mycoplasmas (19\%), mixed infections (8\%), fungi (8\%), and bacteria (4\%) [14]. Bacterial and fungal contamination are usually visible to the unaided eye because of rapid-onset turbidity and culture medium color changes. Standard light microscopy can reveal bacterial cells and fungal structures, thus daily microscopic observation will help early detection of microbial contamination.

This study has several strengths. First, this is the first study to investigate the risk factors for initial fungal contamination during endoscopic biopsy-derived primary ${ }_{3} \mathrm{D}$ culture from non-sterile gastrointestinal track. The results provide valuable guidance to investigators who perform endoscopic biopsy-derived primary $3 \mathrm{D}$ culture, to avoid initial contamination and to increase primary 3D culture yield. Second, we demonstrated various patterns of initial fungal contamination, including unusual bizarre patterns. Serial pattern of bizarre growth reflecting unusual patterns of initial fungal contamination will eventually allow for the early recognition of this pattern of contamination by researchers.

In conclusion, moderate to thick whitish plaques on primary tumors and luminal food retention are associated with initial fungal contamination during biopsy-derived cancer organoid culture. These results suggest that we should exclude the patients whose primary tumors are covered with moderate to thick whitish plaques and those in whom luminal food retention is observed, to avoid fungal contamination and to increase the yield of primary tumor $3 \mathrm{D}$ culture. Most initial fungal contamination can be detected within 3 to 6 days, but unusual initial fungal contamination may be recognized after several passages. Therefore, if unusual growth patterns are observed or if contamination is suspected, researchers should try to identify the cause of contamination as soon as possible.

\section{KEY MESSAGE}

1. Moderate to thick whitish plaques and food retention are clinical risk factors for initial fungal contamination during biopsy-derived cancer organoid culture.

2. Most initial fungal contamination was detected within 3 days, but it could be unusually recognized after several passages.

3. Mild media contamination with preserved organoid can be salvaged. 


\section{Conflict of interest}

No potential conflict of interest relevant to this article was reported.

\section{Acknowledgments}

This project was supported by a grant from the National Research Foundation, Republic of Korea (NRF2015R1DiA1Ao1059219,2019R1FiA1063990, SuYounNam) and Biomedical Research Institute grant, Kyungpook National University Hospital (2016, Su Youn Nam).

\section{REFERENCES}

1. Ridky TW, Chow JM, Wong DJ, Khavari PA. Invasive three-dimensional organotypic neoplasia from multiple normal human epithelia. Nat Med 2010;16:1450-1455.

2. Lancaster MA, Knoblich JA. Organogenesis in a dish: modeling development and disease using organoid technologies. Science 2014;345:1247125.

3. van de Wetering M, Francies HE, Francis JM, et al. Prospective derivation of a living organoid biobank of colorectal cancer patients. Cell 2015;161:933-945.

4. Huang L, Holtzinger A, Jagan I, et al. Ductal pancreatic cancer modeling and drug screening using human pluripotent stem cell- and patient-derived tumor organoids. Nat Med 2015;21:1364-1371.

5. Li X, Francies HE, Secrier M, et al. Organoid cultures recapitulate esophageal adenocarcinoma heterogeneity providing a model for clonality studies and precision therapeutics. Nat Commun 2018;9:2983.

6. Thompson DS, Carlisle PL, Kadosh D. Coevolution of morphology and virulence in Candida species. Eukaryot Cell 2011;10:1173-1182.

7. Kondo J, Endo H, Okuyama H, et al. Retaining cell-cell contact enables preparation and culture of spheroids composed of pure primary cancer cells from colorectal cancer. Proc Natl Acad Sci U S A 2011;108:6235-6240.

8. Kumamoto CA, Vinces MD. Contributions of hyphae and hypha-co-regulated genes to Candida albicans virulence. Cell Microbiol 2005;7:1546-1554.

9. San-Blas G, Travassos LR, Fries BC, et al. Fungal morphogenesis and virulence. Med Mycol 2000;38 Suppl 1:79-86.

10. Sudbery P, Gow N, Berman J. The distinct morphogenic states of Candida albicans. Trends Microbiol 2004;12:317324 .

11. Odds FC. Pathogenesis of Candida infections. J Am Acad Dermatol 1994;31(3 Pt 2):S2-S5.

12. Kumamoto CA, Vinces MD. Alternative Candida albicans lifestyles: growth on surfaces. Annu Rev Microbiol 2005:59:113-133.

13. Mitchell AP. Dimorphism and virulence in Candida albicans. Curr Opin Microbiol 1998;1:687-692.

14. Mirjalili A, Parmoor E, Moradi Bidhendi S, Sarkari B. Microbial contamination of cell cultures: a 2 years study. Biologicals 2005;33:81-85. 
KJIM'

The Korean Journal of Internal Medicine Vol. 36, No. 4, July 2021

Supplementary Table 1. Baseline characteristics

\begin{tabular}{lc}
\hline Characteristic & $\begin{array}{c}\text { Primary cancer } \\
\text { organoid }(\mathrm{n}=45)\end{array}$ \\
\hline Site of primary cancer site & $15(33.3)$ \\
Gastric cancer & $30(66.7)$ \\
$\quad$ Esophageal cancer & $64.9 \pm 10.5$ \\
Age, yr & $42(93.3)$ \\
Male sex & $9(20.0)$ \\
Diabetes & $10(22.2)$ \\
Hypertension & $1(2.2)$ \\
Heart disease & $23(51.1)$ \\
Current smoker & $26(57.8)$ \\
Current drinker & \\
\hline
\end{tabular}

Values are presented as number (\%) or mean \pm SD. 